\title{
Intrauterine Growth Restriction and Hyperoxia as a Cause of White Matter Injury
}

\author{
Jill L. Chang a ${ }^{\text {Mirrah Bashir }}{ }^{a}$ Christiana Santiago $^{d}$ Kathryn Farrow $^{a}$ \\ Camille Fung $^{b}$ Ashley S. Brown ${ }^{b}$ Robert W. Dettman ${ }^{c}$ Maria L.V. Dizon ${ }^{a}$ \\ ${ }^{a}$ Division of Neonatology, Department of Pediatrics, Northwestern University Feinberg School of Medicine, Chicago, IL, \\ USA; 'bivision of Neonatology, Department of Pediatrics, University of Utah School of Medicine, Salt Lake City, UT, USA; \\ 'Stanley Manne Children's Research Institute, Chicago, IL, USA; ${ }^{d}$ Rosalind Franklin University, Chicago, IL, USA
}

\section{Keywords}

Intrauterine growth restriction - Oligodendrocytes - White matter injury · Hyperoxia

\begin{abstract}
Intrauterine growth restriction (IUGR) is estimated to occur in $5 \%$ of pregnancies, with placental insufficiency being the most common cause in developed countries. While it is known that white matter injury occurs in premature infants, the extent of IUGR on white matter injury is less defined in term infants. We used a novel murine model that utilizes a thromboxane $A_{2}\left(T X A_{2}\right)$ analog (U46619), a potent vasoconstrictor, to induce maternal hypertension and mimic human placental insufficiency-induced IUGR to study the white matter. We also investigated the role of hyperoxia as an additional risk factor for white matter injury, as IUGR infants are at increased risk of respiratory comorbidities leading to increased oxygen exposure. We found that TXA $\mathrm{A}_{2}$ analog-induced IUGR results in white matter injury as demonstrated by altered myelin structure and changes in the oligodendroglial cell/oligodendrocyte population. In addition, our study demonstrates that hyperoxia exposure independently results in white matter perturbation. To our knowledge, this is the first study to report single and combined effects of
\end{abstract}

IUGR with hyperoxia impacting the white matter and motor function. These results draw attention to the need for close monitoring of motor development in IUGR babies following hospital discharge as well as highlighting the importance of limiting, as clinically feasible, the degree of oxygen overexposure to potentially improve motor outcomes in this population of infants.

(c) 2018 S. Karger AG, Basel

\section{Introduction}

Intrauterine growth restriction (IUGR) is defined as a significant reduction in fetal growth resulting in birth weight $<10$ th percentile for gestational age $[1,2]$. It affects approximately $5 \%$ of pregnancies worldwide $[3,4]$ and results in an increased risk of mortality and significant morbidities including cerebral palsy (CP), pulmonary hypertension (PHTN), and bronchopulmonary dysplasia (BPD) $[3,5-8]$. In addition, IUGR infants are often admitted to the neonatal intensive care unit and exposed to therapies, such as supraphysiologic oxygen exposure, that put them at a high risk for additional medical complications.

\section{KARGER}

(c) 2018 S. Karger AG, Basel

E-Mail karger@karger.com

www.karger.com/dne
Dr. Jill Chang

Department of Pediatrics, Northwestern University Feinberg School of Medicine 225 E Chicago Ave, Box 45

Chicago, IL 60611 (USA)

E-Mail Jill.chang@ northwestern.edu 
Uteroplacental insufficiency is the most common cause of IUGR in developed countries [8]. Currently, the predominant methods used to induce IUGR in animal models are maternal caloric and protein restriction and bilateral uterine artery ligation $[5,9,10]$. A new murine model of IUGR has been developed which infuses an analog of thromboxane $\mathrm{A}_{2}\left(\mathrm{TXA}_{2}\right)$, a vasoconstrictor that is overexpressed in mothers whose pregnancies are complicated by hypertension, cigarette-smoking, and poorly controlled diabetes. This model therefore more closely mimics the common human pregnancy complications that lead to uteroplacental insufficiency and fetal growth restriction in developed countries $[8,11-13]$.

As mentioned, IUGR is associated with neurologic morbidities including CP. A number of recent population-based cohort studies showed as much as a 5- to 7 -fold increased risk of developing $\mathrm{CP}$ in growth-restricted infants [6, 14-21]. Previous animal studies of IUGR have found evidence of white matter injury, which likely plays a role in the development of CP in this population $[3,5,9,22-25]$. The underlying molecular mechanisms, however, are still poorly defined. Proposed mechanisms vary across studies and include damage to the mature oligodendrocytes (OLs), the arrest of OL maturation, and delayed or impaired myelination. In addition, the longterm effects of IUGR on myelination have not been wellinvestigated and motor function is poorly characterized.

In addition to $\mathrm{CP}$, growth-restricted infants are at an increased risk of cardiovascular and pulmonary morbidities including BPD and PHTN [26-28]. To provide appropriate support of their cardiorespiratory status, these infants are often exposed to supraphysiologic oxygen in the neonatal intensive care unit. Previous studies in murine models have found that hyperoxia exposure alone results in damage to the developing white matter, including ultrastructural changes in myelin, decreased total OLs, and decreased myelin proteins [29-32]. The impact of hyperoxia exposure on the neurodevelopment of IUGR infants has not been evaluated thus far in the literature.

With this novel murine $\mathrm{TXA}_{2}$ model for IUGR, we have shown further evidence that white matter injury occurs following uteroplacental insufficiency-induced growth restriction. Our results show changes in OL subpopulations and altered myelin structure. We also demonstrate changes in white matter integrity and connectivity, and functional motor deficits. In addition, our results support that hyperoxia exposure independently causes white matter injury. Finally, we report that $\mathrm{TXA}_{2}$-induced IUGR in combination with hyperoxia exposure results in more complex injury than exposure to one or the other.

\section{Materials and Methods}

\section{Animals}

All experiments were approved by the Northwestern University Institutional Animal Care and Use Committee. Animal procedures were carried out in accordance with the National Institutes of Health Guide for the Care and Use of Laboratory Animals. Wild-type C57BL/6 mice were purchased from Charles River, Wilmington, MA, USA.

\section{Surgery}

Micro-osmotic pumps (model 1007D, $0.5 \mathrm{~mL} / \mathrm{h}$ ) (Alzet, Cupertino, CA, USA) were implanted in gravid C57Bl/6 wild-type mice at 12.5 days after coitus, correlating with the beginning of the 3rd trimester of human pregnancy. Pumps were inserted into a subcutaneous pocket created in the hip space. The pumps contained either the $\mathrm{TXA}_{2}$-analog U-46619 (catalog No. 16450, Cayman Chemical, Ann Arbor, MI, USA) dissolved in 0.5\% ethanol, or $0.5 \%$ ethanol (vehicle) which was continuously infused at 2,000 $\mathrm{ng} / \mathrm{h}$ throughout the remainder of the pregnancy [8]. Previous model characterization has shown that plasma 11-dehydrothromboxane $\mathrm{B}_{2}$ levels in vehicle- and U-46619-exposed fetuses are similar, providing evidence that U-46619 does not cross the placenta to affect the pups directly.

Pups were delivered spontaneously and were weighed at birth. Pups born to dams receiving $\mathrm{TXA}_{2}$ analog and weighing $<1.266 \mathrm{~g}$, i.e., $<10$ th percentile for weight based on sham pup weights [8], were assigned to the IUGR group. Using this cut-off, approximately one-third of the $\mathrm{TXA}_{2}$-analog pups were defined as small-forgestational age (SGA), which is similar to the incidence of human SGA infants born to mothers with uteroplacental insufficiency in IUGR epidemiological studies [33, 34]. Pups born to dams receiving $0.5 \%$ ethanol and weighing $>1.266 \mathrm{~g}$ ( $>10$ th percentile) were assigned to the vehicle group. Litter sizes were unaltered between groups. All pups were cross-fostered to unmanipulated mouse dams to minimize the surgical effects of pump insertion.

\section{Hyperoxia Exposure}

Litters of vehicle and IUGR pups were placed in either $75 \%$ oxygen (hyperoxia) in a Plexiglass chamber (Biospherix, Lacona, NY, USA) or $21 \%$ oxygen (room air) within $24 \mathrm{~h}$ after birth for 14 days $[35,36]$. Exposure to hyperoxia was continuous, with brief interruptions only for animal care $(<10 \mathrm{~min} /$ day $)$. The concentration of oxygen was maintained with an oxygen controller (ProOx, Biospherix). Ventilation within the chamber was adjusted to remove $\mathrm{CO}_{2}$ such that it did not exceed $0.5 \%$. A hygro-thermometer was used in the chamber to monitor temperature and humidity. The temperature in the chamber did not exceed $23^{\circ} \mathrm{C}$ and the humidity level was maintained by using dishes of desiccant in the bottom of the chamber. A foster dam was placed in the hyperoxia chamber with each vehicle or IUGR litter, and the foster dams were rotated from hyperoxia to room air every $24-48 \mathrm{~h}$ to prevent excessive oxygen toxicity to the adult animals. The litters were removed from the hyperoxia chamber at 14 days and allowed to recover in room air until day of life 28 . The pups continued to be fostered until being weaned at 21 days.

\section{Immunofluorescence}

Mice were anesthetized with 3\% isofluorane, transcardially perfused with $0.9 \% \mathrm{NaCl}$ followed by $4 \%$ paraformaldehyde (PFA) 
in PBS on postnatal day 14 (P14) or P28. Brains were removed, postfixed overnight in ice-cold $4 \%$ PFA, cryoprotected in 30\% sucrose in PBS, embedded and frozen in OCT compound (Sakura Finetek 4583), cut into $10-\mu \mathrm{m}$ sections using a Leica cryostat, and immunostained using the following antibodies: OLIG1 1:600 (Chemicon AB5540) (EMD Millipore, Billirica, MA, USA), OLIG2 1:400 (Chemicon AB9610), CC1 1:100 (Calbiochem APC Ab-7 monoclonal antibody) (EMD Chemicals, San Diego, CA, USA), PDGFRa 1:250 (R\&D Systems AF1062) (Bio-Techne Corp., Minneapolis, MN, USA), AlexaFluor 488 goat anti-rabbit 1:500 (Invitrogen) (ThermoFisher Scientific, Waltham, MA, USA), AlexaFluor 488 goat anti-mouse IgG2b 1:500 (Invitrogen), and AlexaFluor 488 donkey anti-goat 1:500 (Invitrogen). Fields $(\times 40)$ were captured of the corpus callosum dorsomedial to the lateral ventricle using Axiovision 4.6 software (Carl Zeiss Vision, Thornwood, NY, USA). Image files were encoded to mask the study group, areas were measured, and cells were counted using Axiovision 4.6 and ImageJ software (NIH) by investigators blinded to the treatment group. After counts were completed, data were decoded, and cell counts per area were calculated for each field and averaged.

Transmission Electron Microscopy

Samples were obtained on P28 from the internal capsule and fixed in $2 \%$ PFA and $2.5 \%$ glutaraldehyde in $0.1 \mathrm{M}$ sodium cacodylate buffer, $\mathrm{pH}$ 7.3. Samples were processed by the Center of Advanced Microscopy at the Northwestern University Feinberg School of Medicine. They were postfixed with unbuffered 2\% osmium tetroxide, en-bloc stained with $3 \%$ uranyl acetate, rinsed with distilled water, dehydrated in ascending grades of ethanol, transitioned with propylene oxide, embedded in a resin mixture of EMbed-812 kit, and cured in a $60^{\circ} \mathrm{C}$ oven. Samples were sectioned on a Leica EM UC6 ultramicrotome. Sections $(1 \mu \mathrm{m})$ were collected and stained with toluidine blue $\mathrm{O}$ and 70 -nm sections were collected on Formvar carbon-coated film on slotted grids. Thin sections were stained with uranyl acetate and Reynolds lead citrate, and then examined on a transmission electron microscopy (TEM) device, the FEI Tecnai G2 Spirit. Digital images were captured with an FEI Eagle camera at $\times 6,800$ and $\times 23,000$ magnification.

\section{Magnetic Resonance Imaging}

Magnetization transfer imaging (MTR), 3-dimensional gradient recalled echo imaging (3D GRE), and diffusion imaging were performed by 7-T MRI in vivo for all mice on P28. MTR was included for detection of the relative proportion of immobile protons bound to macromolecules and free mobile protons. MTR probed neuronal damage by reflecting regional areas with abnormal brain tissue. 3D GRE provided high-resolution anatomical reference images. Diffusion imaging was incorporated for the detection of regions with abnormal connectivity. From diffusion imaging, the apparent diffusion coefficient (ADC) and fractional anisotropy (FA) were extracted. FA and ADC parametric maps were coregistered to the higher-resolution 3D GRE image. VivoQuant $3 \mathrm{D}$ brain atlas software (Invicro, Boston, MA, USA) was used to delineate regions of interest for the medulla, cerebellum, midbrain, pons, cortex, hippocampus, thalamus, hypothalamus, striatum, pallidum, olfactory, corpus callosum, white matter, and ventricles. With diffusion tensor imaging (DTI) tractography, 3D white matter fiber tracking was performed using DSI Studio software (dsistudio.labsolver.org) with a standard FA threshold of 0.2 . White matter tract volume and length were measured in the following regions of interest: anterior commissure, cerebral peduncle, internal capsule, fimbria of hippocampus, cingulum of the corpus callosum.

\section{Gait Analysis}

Gait analysis was performed using the DigiGait system and software (Mouse Specifics, Boston, MA, USA) on P28. Computerized digital footprint images were generated from a high-speed video recording from the ventral aspect of each mouse as it walked on a motorized transparent treadmill (speed $15-17 \mathrm{~cm} / \mathrm{s}$ ). Data on step-cycle dynamics using the left hind limb of each animal were gathered. Braking time, propulsion time, swing time, stride time, stance time, and ataxia coefficient were averaged for the 10-s sessions as previously described [37].

\section{Statistical Analysis}

Results were analyzed using the nonparametric Kruskal-Wallis test to compare the 4 groups. Dunn's test was used for post hoc analysis. Statistical analyses and data graphing were carried out using GraphPad Prism v7.0 (San Diego, CA, USA). All data are expressed with $n$ representing the number of animals and with significance at $p<0.05$.

\section{Results}

\section{IUGR with and without Hyperoxia Results in}

Decreased OL Numbers in the Corpus Callosum

The cells of the oligodendroglial lineage progress through a series of maturational steps to become myelinating OLs, i.e., oligodendrocyte progenitor cells (OPCs), pre-OLs, immature/premyelinating OLs, and mature OLs [22, 31]. Previous IUGR and hyperoxia animal studies have found conflicting changes in the cells of this lineage, some showing decreases in mature OLs and others showing decrease in OPCs [3, 5, 9, 24, 25, 29, 31, 32].

We performed immunofluorescence staining for specific markers of these cells to better understand the effect of IUGR and hyperoxia on OL maturation. We characterized the OL population at P14 and P28 using OLIG2, a transcription factor that promotes the formation of OPCs and OL differentiation, and is present throughout the entire OL lineage; OLIG1, a transcription factor present in immature/premyelinating OLs and mature OLs, and is thought to be important in both maturation and myelin repair; CC1, a specific marker of myelinating OLs; and PDGFR $\alpha$, a cell surface receptor specific for OPCs [38-42].

We noted a significant decrease in OLIG2+ cells (total OLs) at P14 in the corpus callosum in the IUGR normoxia $(p=0.03, n=6-11)$ and IUGR hyperoxia $(p=0.0006, n=$ 6-7) groups compared to the vehicle normoxia group (Fig. 1a). We also found that P14 OLIG1+ cells (premyelinating and mature OLs) were decreased in the corpus cal- 
Fig. 1. IUGR with and without hyperoxia results in decreased OLs in the corpus callosum early in neurodevelopment. a At P14, there was a significant decrease in OLIG2+ cells in the IUGR (TbxRA, $n=10$ ) and IUGR hyperoxia group $\left(\mathrm{TbxO}_{2}, n=7\right)$ compared to the vehicle group (VehRA, $\left.n=6,{ }^{*} p=0.02\right)$. b At P14, there was a significant decrease in OLIG1+ cells in the IUGR group (TbxRA, $n=18$ ) compared to the vehicle group (VehRA, $n=9, * p=$ 0.0001 ), and in the IUGR hyperoxia group $\left(\mathrm{TbxO}_{2}, n=5\right)$ compared to the vehicle group (VehRA, $n=9$ ), but this did not reach statistical significance. c At P14, there was a significant decrease in $\mathrm{CC} 1+$ cells in the IUGR hyperoxia group $\left(\mathrm{TbxO}_{2}\right.$, $n=4)$ compared to the vehicle group (VehRA, $n=4,{ }^{*} p=0.04$ ). d There were no significant differences in PDGFR $\alpha+$ cells in the 4 groups: VehRA $(n=5)$, TbxRA $(n=$ $4), \mathrm{VehO}_{2}(n=5)$, and $\mathrm{TbxO}_{2}(n=4)$.

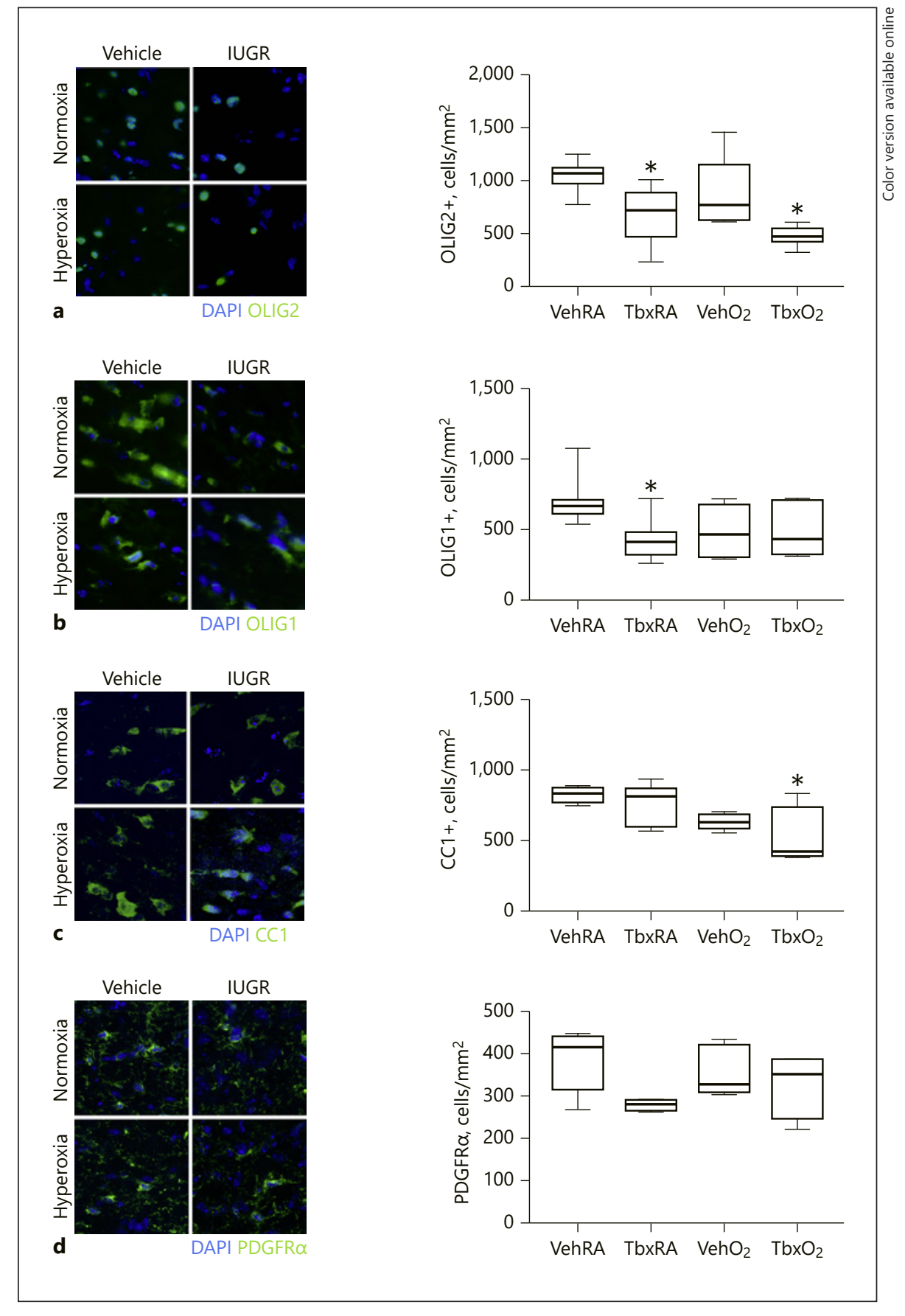

losum in the IUGR normoxia group compared to the vehicle group ( $p=0.002, n=9-18$; Fig. $1 b)$. There was a significant decrease at $\mathrm{P} 14$ in $\mathrm{CC} 1+$ cells (mature myelinating OLs) when IUGR was combined with hyperoxia ( $p=0.04$, $n=4$; Fig. 1c). There were no significant differences inPDGFRa + cells in the 4 groups, i.e., vehicle normoxia $(n=5)$, IUGR normoxia $(n=4)$, vehicle hyperoxia $(n=5)$, and IUGR hyperoxia $(n=4)$ (Fig. 1d). At P28, a decrease in OLIG2+ cells in the corpus callosum was present only in the hyperoxia groups, i.e., vehicle hyperoxia and IUGR with hyperoxia exposure, compared to the vehicle normoxia group ( $p=0.02, n=6-10$; Fig. 2a). There were no significant changes in OLIG1+ cells found at P28 with IUGR or hyperoxia when compared to the vehicle normoxia group (Fig. 2b). There was also a statistically significant decrease in CC1+ and PDGFR $\alpha+$ cells at P28 in the 
Fig. 2. IUGR with hyperoxia results in a persistent decrease in OLs in the corpus callosum in the developed brain. a At P28, there was a significant decrease in OLIG2+ cells in the vehicle hyperoxia $\left(\mathrm{VehO}_{2}, n=\right.$ 6) $(* p=0.01)$ and the IUGR hyperoxia group $\left(\mathrm{TbxO}_{2}, n=6,{ }^{*} p=0.04\right)$ compared to the vehicle group (VehRA, $n=6$ ). $\mathbf{b}$ No significant changes in OLIG1+ cells were found with IUGR or hyperoxia (TbxRA, $\mathrm{VehO}_{2}, \mathrm{TbxO}_{2}, n=5$ ) when compared to the vehicle normoxia group (VehRA, $n=$ 5). c There was a significant decrease in $\mathrm{CC} 1+$ cells in the IUGR hyperoxia group $\left(\mathrm{TbxO}_{2}, n=4\right)$ compared to the vehicle group (VehRA, $n=5, * p=0.01$ ). d There was a significant decrease in PDGFRa+ cells in the IUGR hyperoxia group $\left(\mathrm{TbxO}_{2}\right.$, $n=4)$ compared to the vehicle group (VehRA, $n=4, * p=0.03$ ).

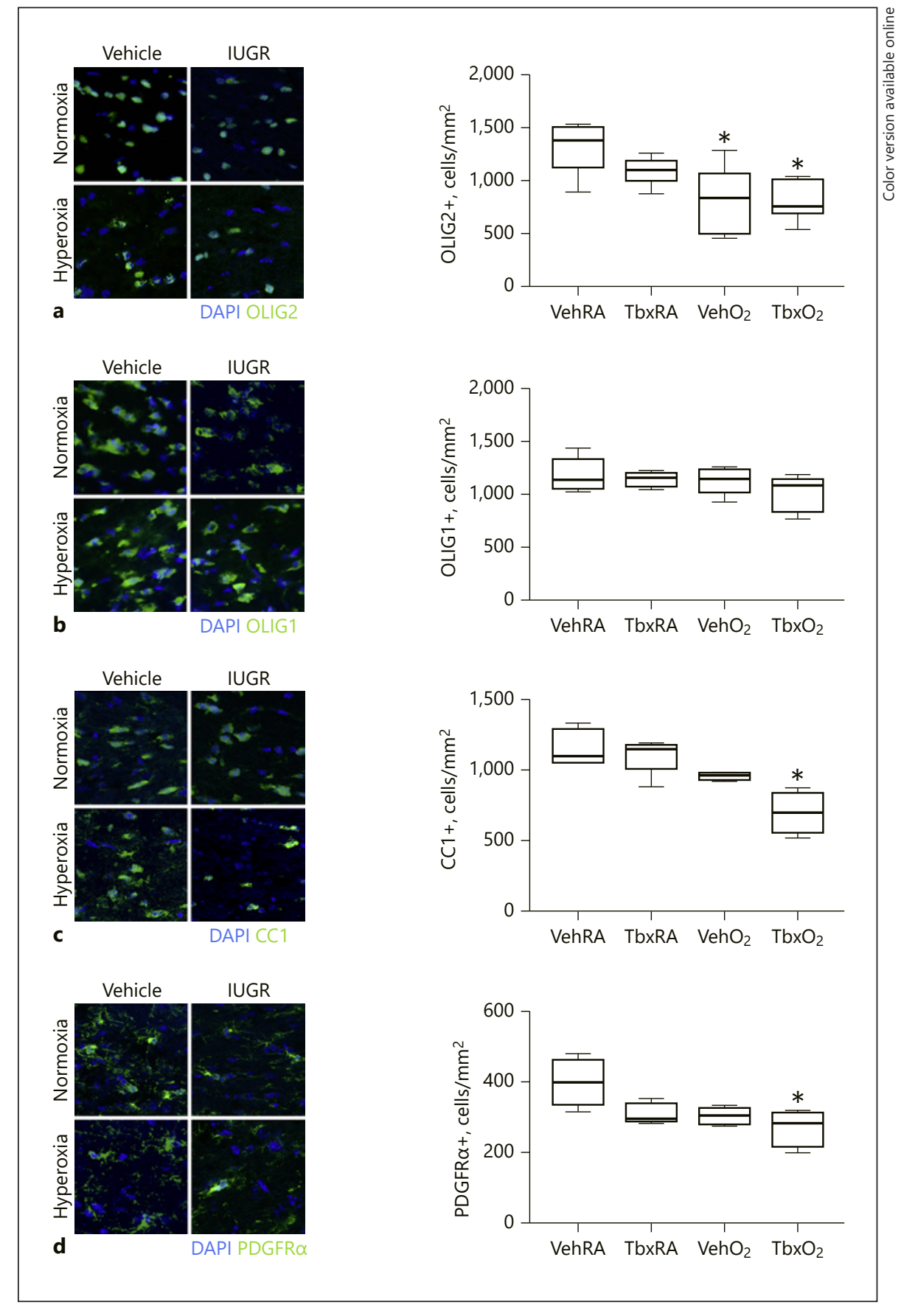

IUGR with hyperoxia group compared to the vehicle normoxia group ( $p=0.01, n=4-5 ; p=0.03, n=4$; Fig. $2 c, d)$.

We therefore conclude that, in early neurodevelopment, IUGR appears to decrease overall OL population with specific decrease in premyelinating OLs, suggesting a limitation in the ability of OPCs to differentiate into premyelinating OLs. At this time, when IUGR is combined with hyperoxia, not only is there a decrease in total
OL population but also specifically in the mature myelinating cells. Additionally, OLIG1 has been shown to be increased in response to postnatal injury, demonstrating its potential importance in the repair of white matter injury. This is supported by studies demonstrating that OLIG1-null mice are severely limited in their ability to repair demyelinated lesions induced by various gliotoxins [39]. Therefore, the fact that IUGR with hyperoxia de- 
Fig. 3. At P14, there was no significant difference in the $0.5 \%$ ethanol vehicle groups with and without hyperoxia (VehRA and $\mathrm{VehO}_{2}$ ) and the normal saline groups (NSRA and $\mathrm{NSO}_{2}$ ) in the number of OLIG $2+$ cells $/ \mathrm{mm}^{2}(n=5-7)(\mathbf{a})$, OLIG $1+$ cells/ $\mathrm{mm}^{2}(n=5-9)(\mathbf{b}), \mathrm{CC} 1+$ cells $/ \mathrm{mm}^{2}$ $(n=4-9)(\mathbf{c})$, and PDGFRa + cells $/ \mathrm{mm}^{2}$ $(n=5-8)(\mathbf{d})$.

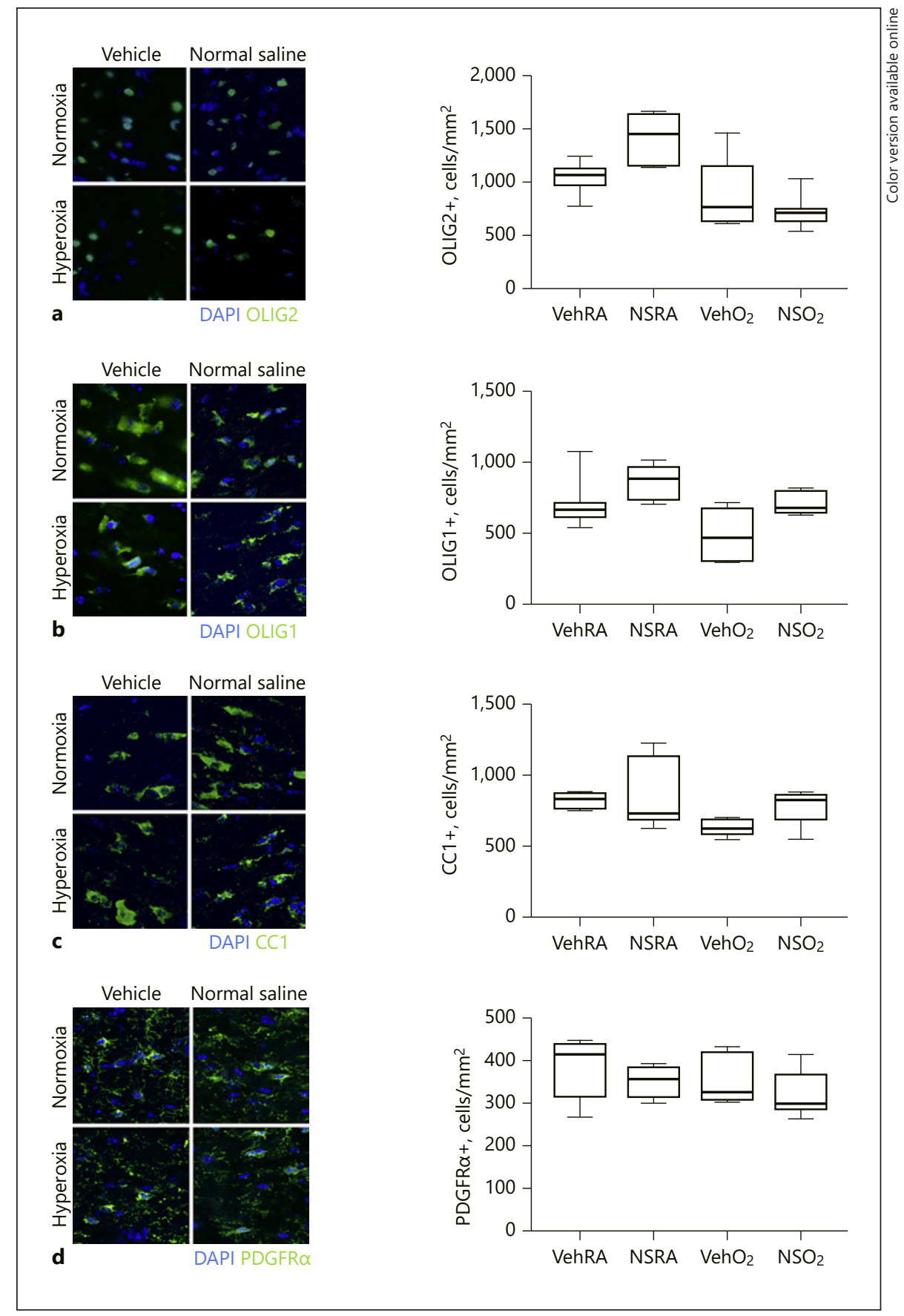

creased $\mathrm{CC} 1+$ cells but had no significant change in OLIG1+ cells can be due to the response to postnatal injury seen with the additional hyperoxia exposure.

This negative effect of hyperoxia was demonstrated again in mature mice by a decrease in total OLs, OPCs, and mature myelinating cells when hyperoxia exposure was combined with IUGR. Again, there was no significant change in OLIG1+ cells despite a decrease in CC1+ cells, potentially in response to this postnatal injury. The lack of statistically significant changes seen at P28 in the IUGR alone group suggests the recovery of premyelinating OLs and therefore total OLs in this group, i.e., a transient effect of IUGR without the additional negative effects of hyperoxia exposure.

Our vehicle group used a $0.5 \%$ ethanol infusion. As ethanol exposure during pregnancy has been shown to 
Fig. 4. At P28, there was no significant difference in the $0.5 \%$ ethanol vehicle groups with and without hyperoxia (VehRA and $\mathrm{VehO}_{2}$ ) and the normal saline groups (NSRA and $\mathrm{NSO}_{2}$ ) in the number of OLIG2+ cells $/ \mathrm{mm}^{2}(n=4-6)(\mathbf{a})$, OLIG1+ cells $/ \mathrm{mm}^{2}(n=4-5)(\mathbf{b}), \mathrm{CC} 1+$ cells $/ \mathrm{mm}^{2}$ $(n=4-5)(\mathbf{c})$, and PDGFRa + cells $/ \mathrm{mm}^{2}$ $(n=4)(\mathbf{d})$.

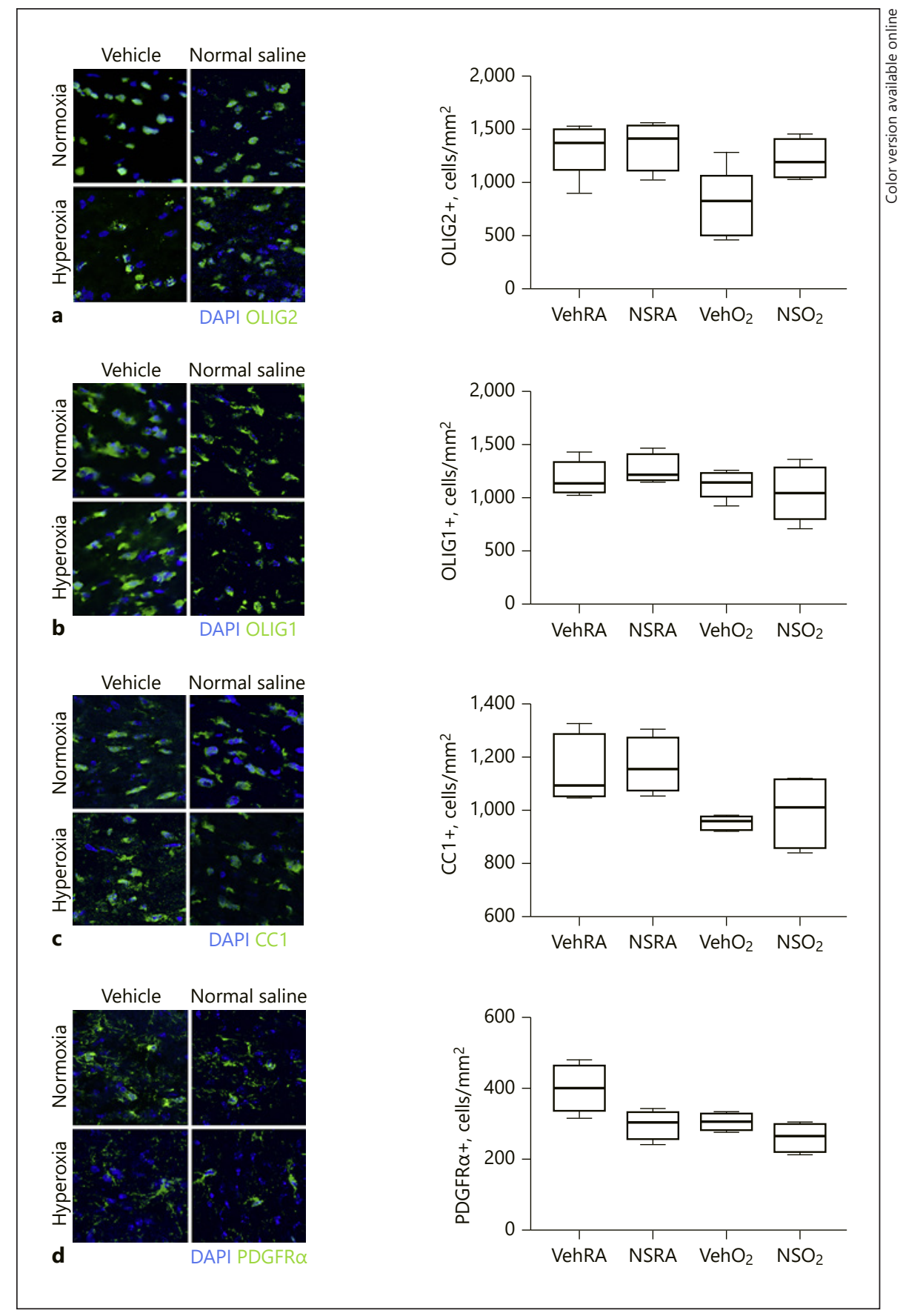

have many negative effects on the developing fetus, we decided to control for this variable by creating a normal saline control. We found no significant differences in any of the OL subpopulations (OLIG2+, OLIG1+, CC1+, and PDGFR $\alpha+$ cells) at P14 or at P28 when the normal saline group was compared to the vehicle $0.5 \%$ ethanol group. This was true also at P14 and P28 in the setting of normoxia and hyperoxia (Fig. 3, 4). Given the lack of signifi- cant differences seen here, our remaining experiments used $0.5 \%$ ethanol as the vehicle control.

To further support our vehicle group not having an effect on our results is that prior studies demonstrated negative neurodevelopmental effects with prenatal ethanol exposure in mouse models using two $2.9 \mathrm{~g} / \mathrm{kg}$ injections of ethanol $4 \mathrm{~h}$ apart, $3 \mathrm{~g} / \mathrm{kg}$ injections daily, and oral ingestion of $25 \%$ ethanol ad lib of dams. In our experiment, 
Fig. 5. IUGR and hyperoxia cause changes in myelin microstructure in the internal capsule at P28. a Myelin structure from the internal capsule of P28 mice was examined by TEM. $\times 6,800$. b An increase in g-ratio was found in the IUGR (TbxRA, $n=7$, * $p=0.004)$ and IUGR with hyperoxia $\left(\mathrm{TbxO}_{2}, n=3,{ }^{*} p=0.01\right)$ groups compared to the vehicle group (VehRA, $n=6$ ). c There was no significant difference in axon diameter in the experimental groups.

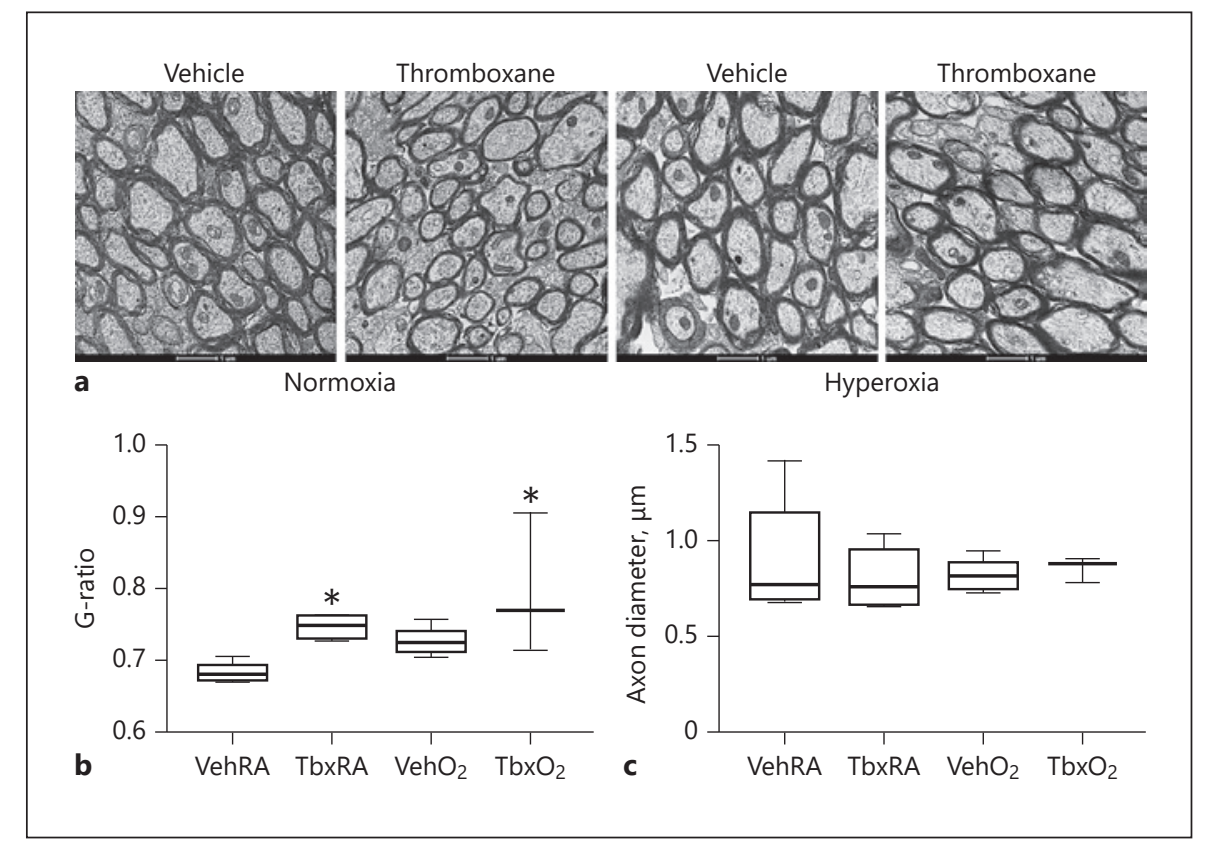

a total of $315.6 \mu \mathrm{g}$ of ethanol was infused over 8 days (39.45 mg/day). Estimating a dam weight of $30 \mathrm{~g}$ at the time of osmotic pump implantation, this is approximately $1.315 \mathrm{mg} / \mathrm{kg} / \mathrm{day}$, which is far lower than the amount of ethanol used in these studies [43-45].

\section{IUGR with and without Hyperoxia Results in Changes in Myelin Structure}

Given the changes in OLs, we next investigated changes in myelination with IUGR and hyperoxia exposure. Myelin structure from the internal capsule of P28 mice from all 4 groups was examined by TEM (Fig. 5a). The internal capsule was examined, as the corticospinal tract (CST) constitutes a large portion of the internal capsule and is integral in carrying motor information from the primary motor cortex to the lower motor neurons in the spinal cord. The CST has also been implicated in the motor dysfunction seen in CP. Myelin thickness, g-ratio, and axon diameter were compared. G-ratio is the ratio of the inner axonal diameter to the total outer diameter [46, 47]. Assuming a constant axonal diameter, an increased g-ratio suggests decreased myelin thickness. We observed an increase in g-ratio with IUGR alone ( $p=0.004, n=7)$ and when IUGR was combined with hyperoxia ( $p=0.01, n=4)$ compared to the vehicle group $(n=6)$ (Fig. 5b). There were no significant changes in axon diameter between groups (Fig. 5c). The increase in g-ratio with unchanged axon diameter found with IUGR and IUGR combined with hyperoxia indi- cates a significant decrease in myelin thickness in these groups.

\section{IUGR and Hyperoxia Result in Changes in White Matter Integrity and Tracts}

With these changes in myelin thickness, we also wanted to determine if white matter integrity was affected, so DTIMRI was performed. DTI assesses the directionality of the random motion of water molecules within biologic tissue and has been shown to have high sensitivity for changes in myelination and white matter organization, both during development and after injury [31, 48-50]. Decreased FA and increased ADC have been found with white matter injury in the preterm population and with hyperoxia exposure $[31,50,51]$.

Diffusion imaging performed at P28 demonstrated a significant decrease in FA in the midbrain $(p=0.01)$ and pons $(p=0.006)$ in the vehicle hyperoxia group compared to the vehicle normoxia group $(n=7)$ (Fig. 6a). When IUGR was combined with hyperoxia exposure $(n=4)$, there was a significant decrease in FA seen in the striatum $(p=0.01)$, pallidum $(p=0.005)$, and cerebellum $(p=0.05)$ (Fig. 6b). Thus, decreased FA values were found with hyperoxia alone and when IUGR was combined with hyperoxia in different areas of the brain. With hyperoxia exposure, white matter integrity was affected within the structures of the brainstem (midbrain and pons) that are important for both motor function and balance. With IUGR combined with hyperoxia, the white matter integ- 
rity of the structures integral for regulating motor movements (the striatum, pallidum, and cerebellum) were particularly affected.

Fiber tractography was used to measure white matter tract volume and length in the cerebral peduncle, fimbria of the hippocampus, internal capsule, and cingulum of the corpus callosum. Hyperoxia alone significantly decreased white matter tract volume in the cerebral peduncles $(p=0.01, n=7-8)$ (Fig. 7a), fimbria of the hippocampus $(p=0.02)($ Fig. $3 b)$, and internal capsule $(p=0.01)$
Fig. 6. Hyperoxia alone and IUGR with hyperoxia result in changes in myelin integrity as seen with DTI MRI at P28. a Significant decreases in FA in the midbrain ${ }^{*} p=0.01$ and pons $(* p=0.006)$ were seen in the hyperoxia group $\left(\mathrm{VehO}_{2}, n=7\right)$ compared to the vehicle normoxia group (VehRA, $n=7$ ). b IUGR with hyperoxia exposure group $\left(\mathrm{TbxO}_{2}, n=4\right)$ showed significant decreases in FA in the striatum $(* p=0.01)$, pallidum $\left(^{*} p=0.005\right)$, and cerebellum $(* p=0.05)$ when compared to the vehicle group (VehRA, $n=7$ ).

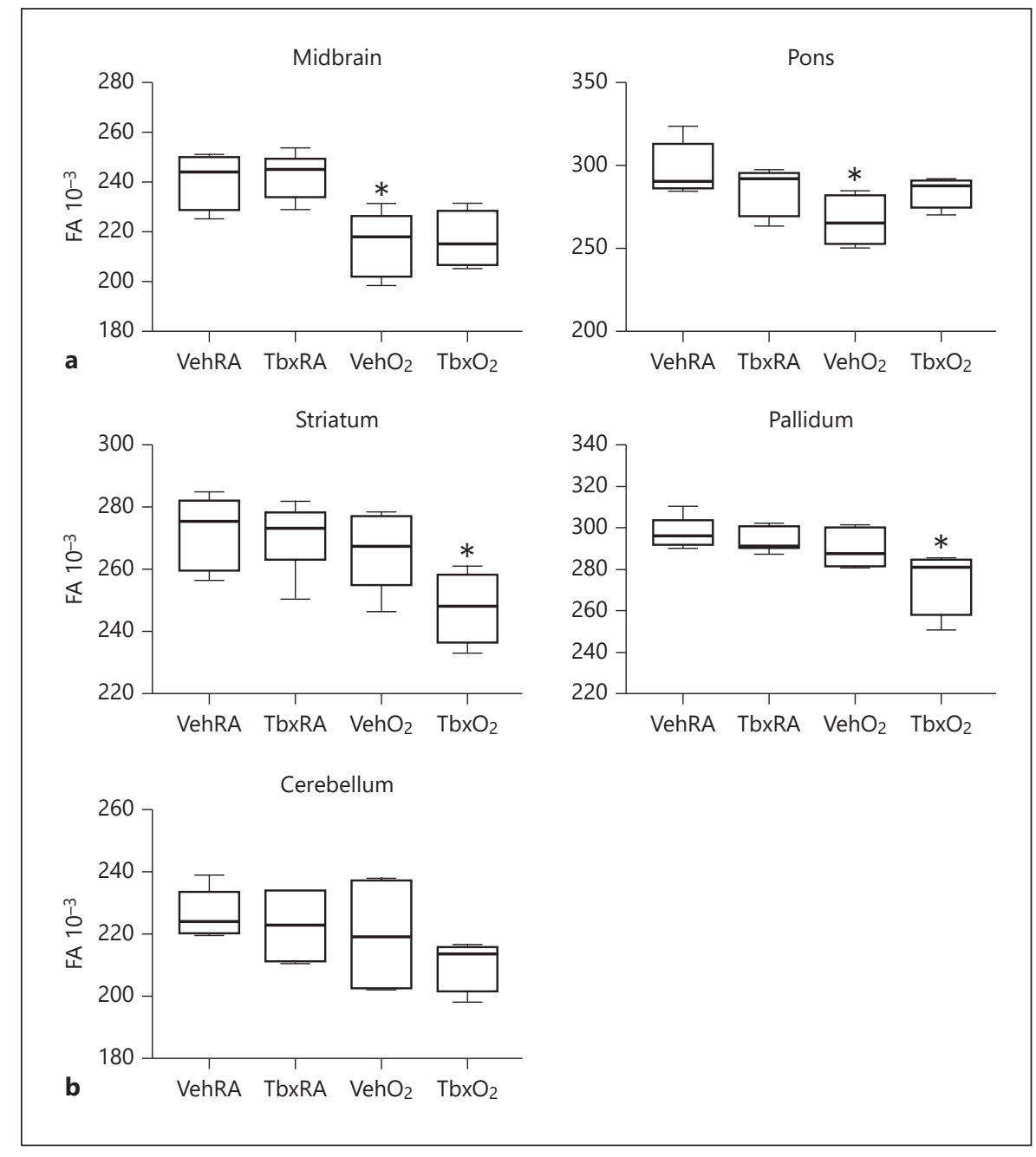

Fig. 7. Fiber tractography showed changes in white matter tract length and/or volume in the cerebral peduncle, fimbria of hippocampus, internal capsule, and cingulum of the corpus callosum. a Tract volume was significantly decreased in the cerebral peduncle with hyperoxia exposure alone $\left(\mathrm{VehO}_{2}, n=7, * p=0.01\right)$ compared to the vehicle group (VehRA, $n=7$ ). Coronal section showing white matter fiber tracts $\left({ }^{* *}\right)$ through the cerebral peduncle (red). b Tract volume was significantly decreased in the fimbria of the hippocampus in the vehicle hyperoxia $\left(\mathrm{VehO}_{2}, n=7,{ }^{*} p=0.02\right)$ and IUGR with hyperoxia $\left(\mathrm{TbxO}_{2}, n=4,{ }^{*} p=0.04\right)$ groups compared to the vehicle group (VehRA, $n=8$ ). Coronal section showing white matter fiber tracts through the fimbria of the hippocampus (orange). c White matter tract volume was significantly decreased with IUGR (TbxRA, $n=7$,
$* p=0.01)$, hyperoxia exposure alone $\left(\mathrm{VehO}_{2}, n=7, * p=0.01\right)$, and IUGR with hyperoxia $\left(\mathrm{TbxO}_{2}, n=4,{ }^{*} p<0.0001\right)$ compared to the vehicle group (VehRA, $n=7$ ). Tract length was also significantly decreased with hyperoxia exposure alone $\left(\mathrm{VehO}_{2}, n=7,{ }^{*} p=0.04\right)$ and IUGR with hyperoxia $\left(\mathrm{TbxO}_{2}, n=4,{ }^{*} p=0.01\right)$. Coronal section showing white matter fiber tracts through the internal capsule (blue). d White matter tract volume was significantly decreased in the cingulum of the corpus callosum in IUGR with hyperoxia $\left(\mathrm{TbxO}_{2}, n=\right.$ 4) compared to vehicle normoxia (VehRA, $n=8$, ${ }^{*} p=0.04$ ) (see Fig. $3 d$ ). Coronal section showing white matter fiber tracts through the cingulum of the corpus callosum (yellow). ${ }^{* *}$ For the fiber tracts, the convention used for directional color mapping was red for leftright, green for anteroposterior, and blue for superior-inferior.

(For figure see next page.) 

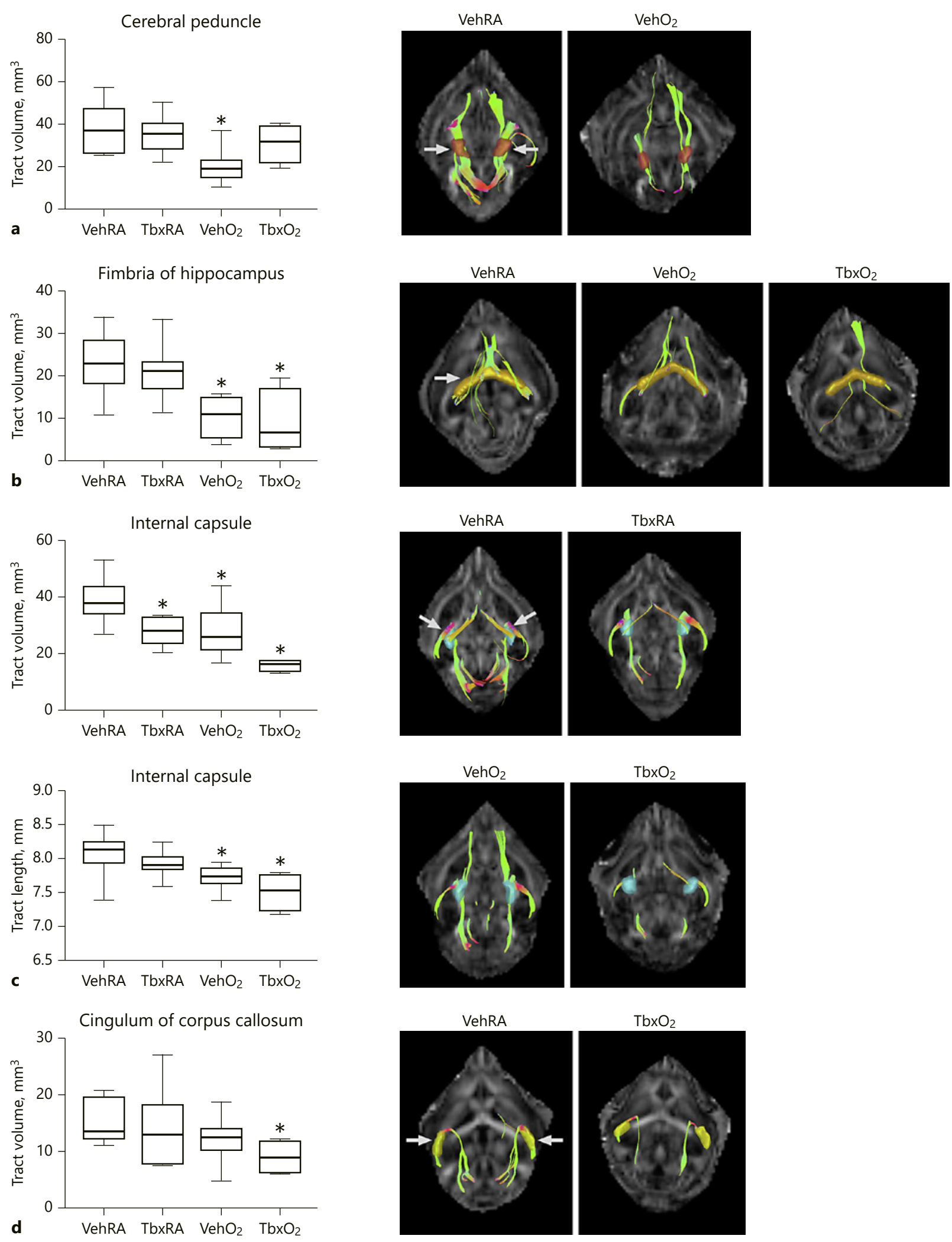


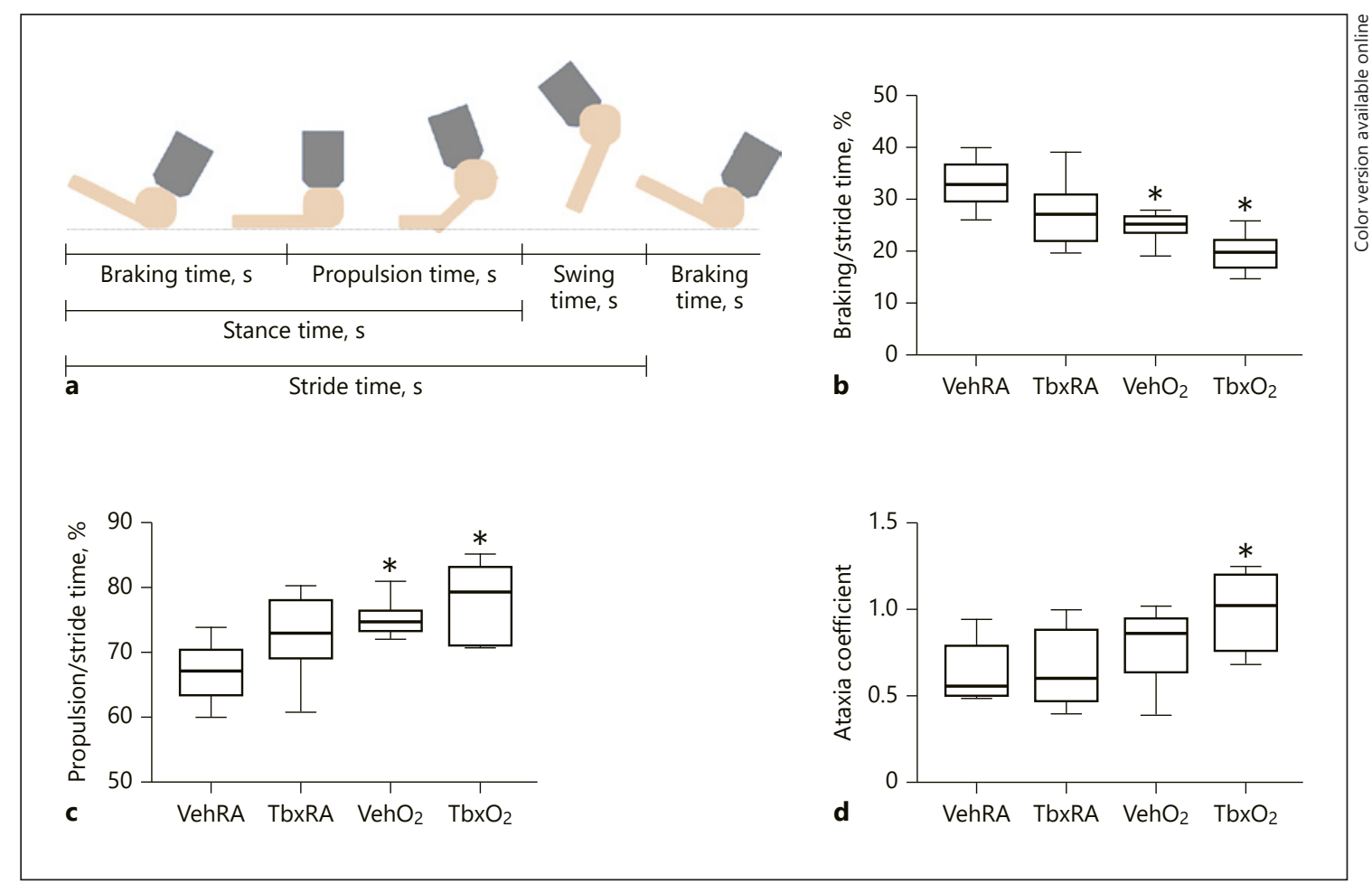

Fig. 8. IUGR and hyperoxia result in functional motor deficits. Gait parameters were evaluated using the Mouse Specifics DigiGait system. Differences in gait were seen at P28 at a speed of 15$17 \mathrm{~cm} / \mathrm{s}$. a Five main components of gait were analyzed: braking, propulsion, swing, stance, and stride time. b Decreased percent braking/stride time was seen with IUGR (TbxRA, $n=11$ ), hyperoxia exposure $\left(\mathrm{VehO}_{2}, n=10, * p=0.08\right)$, and IUGR combined with hyperoxia $\left(\mathrm{TbxO}_{2}, n=6,{ }^{*} p<0.0001\right)$ compared to the vehicle group (VehRA, $n=11$ ). c Increased percent propulsionl/ stride time was seen with IUGR (TbxRA, $n=11$ ), hyperoxia exposure $\left(\mathrm{VehO}_{2}, n=10,{ }^{*} p=0.002\right)$, and IUGR combined with hyperoxia $\left(\mathrm{TbxO}_{2}, n=6,{ }^{* *} p=0.002\right)$ compared to the vehicle group (VehRA, $n=11)$. d Increased ataxia coefficients ( $\left.{ }^{*} p=0.02\right)$ were found with IUGR with hyperoxia exposure $\left(\mathrm{TbxO}_{2}, n=6\right)$ compared to the vehicle group (VehRA, $n=11$ ). Note: total stance and stride times were not significantly different between vehicle group and IUGR, hyperoxia, or IUGR with hyperoxia. compared to the vehicle normoxia group (Fig. 7c). Hyperoxia exposure alone significantly decreased white matter tract length in the internal capsule with hyperoxia $(p=0.04)$. While trends toward decreased white matter tract volume with IUGR exposure alone was seen in multiple areas of the brain, it was only found to be statistically significant in the internal capsule when compared to the vehicle normoxia group ( $p=0.01, n=7-8$; Fig. 7c). When IUGR was combined with hyperoxia, not only were the internal capsule tract volume $(p<0.0001)$ and length $(p=0.01)$ decreased (Fig. 7c), but there were also decreases in white matter volume in the fimbria of the hippocampus ( $p=0.04)$ (Fig. $7 \mathrm{~b})$ and the cingulum of the corpus callosum $(p=0.04)$ compared to the vehicle normoxia group (Fig. $7 d$ ). In summary, the white matter tract volume in the internal capsule was significantly decreased in all 3 experimental groups compared to the vehicle normoxia group. Hyperoxia exposure alone and in combina- tion with IUGR resulted in decreased white matter tract volume in multiple areas of the brain.

\section{Exposure to IUGR and Hyperoxia Results in \\ Functional Gait Motor Deficits}

Gait analysis was used as a quantitative measure to determine if motor function was affected with IUGR and hyperoxia. DigiGait analysis performed on P28 mice demonstrated changes in gait parameters (Fig. 8a) at a speed of $15-17 \mathrm{~cm} / \mathrm{s}$. The hyperoxia $(n=10)$ and IUGR with hyperoxia $(n=6)$ groups both showed a decrease in percent braking time (defined as the amount of time from the first contact with the treadmill to the time of peak stance) per stride compared to the vehicle normoxia group (Fig. 8b) (hyperoxia group, $p=0.08$; IUGR with hyperoxia group, $p<0.0001)$. Similarly, both groups showed an increase in percent propel time (the moment of peak stance through full lifting of the paw off the belt) 
of each stride compared to the vehicle normoxia group (Fig. 8c) (hyperoxia group, $p=0.002$; IUGR with hyperoxia group, $p=0.002$ ). Additional evidence of gait disturbance including increased ataxia coefficient (a measure of step-to-step variability; $p=0.02$ ) was seen in the IUGR group exposed to hyperoxia (Fig. 8d). Total stance and stride time did not differ with IUGR, hyperoxia, or IUGR with hyperoxia. In summary, alterations in gait, indicating motor dysfunction, were seen with IUGR and hyperoxia exposure.

\section{Discussion}

Our study is the first to use a novel TXA $\mathrm{T}_{2}$-analog murine model to investigate how uteroplacental insufficiency-induced IUGR would affect white matter composition and function. We demonstrated white matter injury as changes in the OL population, myelin integrity and structure, white matter tracts, and gait. We are also the first to investigate the impact of hyperoxia exposure on the development of the white matter in IUGR mice. Our findings provide insight into why certain growth-restricted babies go on to develop motor dysfunction and are at a higher risk of CP.

With this TXA $_{2}$-analog model of IUGR, we found a decrease in total OL population, specifically immature/ premyelinating OLs with IUGR in early neurodevelopment. We postulate that this may be attributed to a limitation in the ability of OPCs to differentiate into premyelinating OLs following IUGR. This is supported by previous studies that have found early OLs to be more sensitive to apoptosis in models of prematurity and hypoxic-ischemic injury [52]. There is recovery of premyelinating OLs and total OLs by adulthood, suggesting a transient effect of IUGR on differentiation. Although the changes in OL populations appear transient and recover by adulthood, we found that IUGR resulted in a decrease in myelin thickness in the internal capsule and a decrease in white matter tract volume in the internal capsule on MRI. This suggests a long-term effect on myelin production independent of the number of mature myelinating cells, and it warrants further investigation.

We were most intrigued by our combined insult of IUGR and hyperoxia, which appears to elicit a more complex and persistent effect on the white matter. With the addition of hyperoxia to IUGR, there is an early decrease in total OLs and mature myelinating cells, that, unlike IUGR alone, persists to adulthood, thereby indicating chronic injury. There is also a late decrease in OPCs in

White Matter Injury with IUGR and Hyperoxia adulthood, that may be the result of decreased proliferation, apoptosis, or increased migration out of the corpus callosum. Additionally, our MRI studies demonstrated reduced myelin integrity and white matter tract volume and length in multiple areas of the brain. We believe that this is potentially a result of abnormal myelin sheath formation and/or thinner myelin sheaths due to a disturbance of the mature myelinating OLs. Additionally, we speculate that the myelination defect likely results in the gait changes detected in our mice with IUGR and hyperoxia. Gait changes are of great clinical significance as children with $\mathrm{CP}$ are known to have significant perturbations in movement and posture which impair their activities of daily living. In fact, the diagnosis of $\mathrm{CP}$ is made based on the observation of abnormal muscle tone or posture and the presence of gait abnormalities in young children $[53,54]$.

Our study also demonstrates that hyperoxia exposure independently results in white matter dysfunction and appears to affect white matter differently from IUGR. In contrast to changes in OL populations and myelin thickness as seen with IUGR, hyperoxia resulted in impaired myelin integrity and decreased white matter tract volume, seen on MRI. We believe that this is potentially a result of abnormal myelin sheath formation and/or thinner myelin sheaths due to a decrease in mature myelinating OLs.

The areas of the brain affected by hyperoxia exposure alone differed from those affected when hyperoxia was combined with IUGR. One explanation for this is the difference in timing of injury with IUGR, which occurs prenatally from decreased placental blood flow to the fetus, while hyperoxia exposure occurs in the postnatal period. In rodents, late gestation correlates to a time of oligodendrogenesis and where OPCs and immature OLs predominate [55]. At P7, OL maturation is evident along with the onset of early myelin maturation [52]. Additionally, at $\mathrm{P} 2-\mathrm{P} 5$, the CST of rodents has reached the spinal cord and has just begun innervating the gray matter [55]. Therefore, it is not surprising that the timing of an injury can play a significant role in the type of white matter injury seen, and this speaks for the ongoing vulnerability of these IUGR infant brains to postnatal insults.

Finally, our results also showed CST involvement, when IUGR is combined with hyperoxia exposure, observed as changes on DTI in the corpus callosum, internal capsule, and cerebral peduncles. Abnormal CST anatomy has been implicated as a source of the deficits seen in patients with spastic $\mathrm{CP}$, which is the most common type of $\mathrm{CP}$ associated with perinatal injury from IUGR and pre- 
maturity $[6,14,19,20]$. These results lend insight into the pathogenesis of functional motor deficits and studies and interventions targeting the CST in the future are warranted.

In conclusion, we believe this model of IUGR to be clinically and translationally relevant for white matter injury investigations. We have added evidence here that IUGR infants are at high risk of white matter injury, the mechanism of which involves diminution of OLs and impaired myelination. From our results, we postulate that a more complex mechanism of white matter injury involving 2 sequential insults occurs when IUGR is combined with hyperoxia. These findings may explain why certain IUGR infants go on to develop worse motor dysfunction and CP. This is supported by the fact that while neonatal brain injuries might result from antenatal and neonatal insults alone, CP seems to result from postnatal damage to the brain including the distinct complications of prematurity. For instance, premature infants with lung disease such as BPD and congenital heart disease, who are likely chronically hypoxemic, have the highest risk of poor long-term neurodevelopmental outcomes [38]. Few studies at this time have integrated comorbid conditions such as these in animal models of neonatal brain injury.

Despite these intriguing results, we recognize several limitations to our study. First, the compressed developmental time frame of rodent white matter development can only provide an indication of the actual mechanism of human white matter injury. Second, we did not estab- lish any direct cause-and-effect of the changes in OL numbers and decreased myelination on other components of motor function. Additionally, we did not explore signaling pathways that participate in OL fate or myelination after IUGR and/or hyperoxia. Studies examining the molecular mechanisms responsible for these phenotypes are the next step in our investigation and include the downregulation of bone morphogenetic protein signaling in this injury model.

We believe that our results identify a potentially modifiable risk factor, supraphysiologic oxygen exposure, in this high-risk population. In addition, for these high-risk infants, we also believe that a comprehensive neurological exam at term gestation is crucial to identify changes in posture, tone, or motor function. If functional deficits are identified, brain MRI may provide additional benefits by localizing the regions of white matter injury.

\section{Acknowledgements}

Grants supporting this research include Northwestern Memorial Foundation Friends of Prentice Grant Initiative and NINDS R01NS086945. This work was also supported by Northwestern University Center for Advanced Molecular Imaging and the Northwestern University Behavioral Phenotyping Core.

\section{Disclosure Statement}

The authors have no conflicts of interest to declare.

\section{References}

1 Schröder HJ. Models of fetal growth restriction. Eur J Obstet Gynecol Reprod Biol. 2003 Sep;110(suppl 1):S29-39.

2 Battaglia FC, Lubchenco LO. A practical classification of newborn infants by weight and gestational age. J Pediatr. 1967 Aug;71(2): $159-63$.

3 Tolcos M, Bateman E, O’Dowd R, Markwick $\mathrm{R}$, Vrijsen K, Rehn A, et al. Intrauterine growth restriction affects the maturation of myelin. Exp Neurol. 2011 Nov;232(1):53-65.

4 Mandruzzato G, Antsaklis A, Botet F, Chervenak FA, Figueras F, Grunebaum A, et al.; WAPM. Intrauterine restriction (IUGR). J Perinat Med. 2008;36(4):277-81.

5 Reid MV, Murray KA, Marsh ED, Golden JA, Simmons RA, Grinspan JB. Delayed myelination in an intrauterine growth retardation model is mediated by oxidative stress upregulating bone morphogenetic protein 4 . J Neuropathol Exp Neurol. 2012 Jul;71(7):640-53.
6 Jacobsson B, Ahlin K, Francis A, Hagberg G, Hagberg H, Gardosi J. Cerebral palsy and restricted growth status at birth: populationbased case-control study. BJOG. 2008 Sep; 115(10):1250-5.

7 Levine T, Grunau R, McAuliffe F, Pinnamaneni R, Foran A, Alderdice F. Early childhood neurodevelopment after intrauterine growth restriction: a systemic review. Pediatrics. 2015; 135(1): 135.

8 Fung C, Brown A, Cox J, Callaway C, McKnight R, Lane R. Novel thromboxane A2 analog-induced IUGR mouse model. J Dev Orig Health Dis. 2011 Oct;2(5):291-301.

9 Basilious A, Yager J, Fehlings M. Neurological outcomes in animal models of uterine artery ligation and relevance to human intrauterine growth restriction: a systemic review. Dev Med Child Neurol. 2015 May;57(5):420-30.
10 Tolcos M, McDougall A, Shields A, Chung Y, O’Dowd R, Turnley A, et al. Intrauterine Growth Restriction Affects Cerebellar Granule Cells in the Developing Guinea Pig Brain. Dev Neurosci. 2018;40(2):162-74.

11 Hayakawa M, Takemoto K, Nakayama A, Saito A, Sato Y, Hasegawa M, et al. An animal model of intrauterine growth retardation induced by synthetic thromboxane a(2). J Soc Gynecol Investig. 2006 Dec;13(8):566-72.

12 McAdam BF, Byrne D, Morrow JD, Oates JA. Contribution of cyclooxygenase- 2 to elevated biosynthesis of thromboxane $\mathrm{A} 2$ and prostacyclin in cigarette smokers. Circulation. 2005 Aug;112(7):1024-9.

13 Tanbe AF, Khalil RA. Circulating and vascular bioactive factors during hypertension in pregnancy. Curr Bioact Compd. 2010 Mar; 6(1):60-75. 
14 Freire G, Shevell M, Oskoui M. Cerebral palsy: phenotypes and risk factors in term singletons born small for gestational age. Eur J Paediatr Neurol. 2015 Mar;19(2):218-25.

15 Streja E, Miller JE, Wu C, Bech BH, Pedersen LH, Schendel DE, et al. Disproportionate fetal growth and the risk for congenital cerebral palsy in singleton births. PLoS One. 2015 May;10(5):e0126743.

16 Mor O, Stavsky M, Yitshak-Sade M, Mastrolia SA, Beer-Weisel R, Rafaeli-Yehudai T, et al. Early onset preeclampsia and cerebral palsy: a double hit model? Am J Obstet Gynecol. 2016 Jan;214(1):105.e1-9.

17 Blair EM, Nelson KB. Fetal growth restriction and risk of cerebral palsy in singletons born after at least 35 weeks' gestation. Am J Obstet Gynecol. 2015 Apr;212(4):520.e1-7.

18 Nelson KB, Blair E. Prenatal Factors in Singletons with Cerebral Palsy Born at or near Term. N Engl J Med. 2015 Sep;373(10):94653.

19 Dahlseng MO, Andersen GL, Irgens LM, Skranes J, Vik T. Risk of cerebral palsy in termborn singletons according to growth status at birth. Dev Med Child Neurol. 2014 Jan;56(1): 53-8.

20 Ahlin K, Himmelmann K, Hagberg G, Kacerovsky $\mathrm{M}$, Cobo $\mathrm{T}$, Wennerholm UB, et al. Non-infectious risk factors for different types of cerebral palsy in term-born babies: a population-based, case-control study. BJOG. 2013 May;120(6):724-31.

21 McIntyre S, Taitz D, Keogh J, Goldsmith S, Badawi N, Blair E. A systematic review of risk factors for cerebral palsy in children born at term in developed countries. Dev Med Child Neurol. 2013 Jun;55(6):499-508.

22 Volpe JJ, Kinney HC, Jensen FE, Rosenberg PA. Reprint of "The developing OL: key cellular target in brain injury in the premature infant". Int J Dev Neurosci. 2011 Oct;29(6): $565-82$.

23 Vuguin PM. Animal models for small for gestational age and fetal programming of adult disease. Horm Res. 2007;68(3):113-23.

24 Olivier P, Baud O, Bouslama M, Evrard P, Gressens P, Verney C. Moderate growth restriction: deleterious and protective effects on white matter damage. Neurobiol Dis. 2007 Apr;26(1):253-63.

25 Rideau Batista Novais A, Pham H, Van De Looij Y, Bernal M, Mairesse J, Zana-Taieb E, et al. Transcriptomic regulations in oligodendroglial and microglial cells related to brain damage following fetal growth restriction. Glia. 2016 Dec;64(12):2306-20.

26 Eriksson L, Haglund B, Odlind V, Altman M, Ewald U, Kieler H. Perinatal conditions related to growth restriction and inflammation are associated with an increased risk of bronchopulmonary dysplasia. Acta Paediatr. 2015 Mar;104(3):259-63.

27 Mestan KK, Check J, Minturn L, Yallapragada S, Farrow KN, Liu X, et al. Placental pathologic changes of maternal vascular underper- fusion in bronchopulmonary dysplasia and pulmonary hypertension. Placenta. 2014 Aug; 35(8):570-4.

28 Rozance PJ, Seedorf GJ, Brown A, Roe G, O'Meara MC, Gien J, et al. Intrauterine growth restriction decreases pulmonary alveolar and vessel growth and causes pulmonary artery endothelial cell dysfunction in vitro in fetal sheep. Am J Physiol Lung Cell Mol Physiol. 2011 Dec;301(6):L860-71.

29 Ritter J, Schmitz T, Chew LJ, Bührer C, Möbius $\mathrm{W}$, Zonouzi M, et al. Neonatal hyperoxia exposure disrupts axon-OL integrity in the subcortical white matter. J Neurosci. 2013 May;33(21):8990-9002.

30 Ramani M, van Groen T, Kadish I, Bulger A, Ambalavanan N. Neurodevelopmental impairment following neonatal hyperoxia in the mouse. Neurobiol Dis. 2013 Feb;50:69-75.

31 Schmitz T, Ritter J, Mueller S, FelderhoffMueser U, Chew LJ, Gallo V. Cellular changes underlying hyperoxia-induced delay of white matter development. J Neurosci. 2011 Mar; 31(11):4327-44.

32 Gerstner B, DeSilva TM, Genz K, Armstrong A, Brehmer F, Neve RL, et al. Hyperoxia causes maturation-dependent cell death in the developing white matter. J Neurosci. 2008 Jan;28(5):1236-45.

33 Eskenazi B, Fenster L, Sidney S, Elkin EP. Fetal growth retardation in infants of multiparous and nulliparous women with preeclampsia. Am J Obstet Gynecol. 1993 Nov;169(5): $1112-8$.

34 Pietrantoni M, O'Brien WF. The current impact of the hypertensive disorders of pregnancy. Clin Exp Hypertens. 1994 Jul;16(4):47992.

35 Aslam M, Baveja R, Liang OD, FernandezGonzalez A, Lee C, Mitsialis SA, et al. Bone marrow stromal cells attenuate lung injury in a murine model of neonatal chronic lung disease. Am J Respir Crit Care Med. 2009 Dec; 180(11):1122-30

36 Lee KJ, Berkelhamer SK, Kim GA, Taylor JM, O'Shea KM, Steinhorn RH, et al. Disrupted pulmonary artery cyclic guanosine monophosphate signaling in mice with hyperoxiainduced pulmonary hypertension. Am J Respir Cell Mol Biol. 2014 Feb;50(2):369-78.

37 Hampton TG, Stasko MR, Kale A, Amende I, Costa AC. Gait dynamics in trisomic mice: quantitative neurological traits of Down syndrome. Physiol Behav. 2004 Sep;82(2-3):381-9.

38 Silbereis JC, Huang EJ, Back SA, Rowitch DH. Towards improved animal models of neonatal white matter injury associated with cerebral palsy. Dis Model Mech. 2010 Nov-Dec; 3(11-12):678-88.

39 Meijer DH, Kane MF, Mehta S, Liu H, Harrington E, Taylor $\mathrm{CM}$, et al. Separated at birth? The functional and molecular divergence of OLIG1 and OLIG2. Nat Rev Neurosci. 2012 Dec;13(12):819-31.

40 Dai J, Bercury KK, Ahrendsen JT, Macklin WB. Olig1 function is required for OL differ- entiation in the mouse brain. J Neurosci. 2015 Mar;35(10):4386-402.

41 Xin M, Yue T, Ma Z, Wu FF, Gow A, Lu QR. Myelinogenesis and axonal recognition by OLs in brain are uncoupled in Olig1null mice. J Neurosci. 2005 Feb;25(6):135465.

42 Jakovcevski I, Filipovic R, Mo Z, Rakic S, Zecevic N. Oligodendrocyte development and the onset of myelination in the human fetal brain. Front Neuroanat. 2009 Jun;3:5

43 Sulik KK, Johnston MC, Webb MA. Fetal alcohol syndrome: embryogenesis in a mouse model. Science. 1981 Nov;214(4523):936-8.

44 Jégou S, El Ghazi F, de Lendeu PK, Marret S, Laudenbach V, Uguen A, et al. Prenatal alcohol exposure affects vasculature development in the neonatal brain. Ann Neurol. 2012 Dec; 72(6):952-60.

45 El Shawa H, Abbott CW 3rd, Huffman KJ. Prenatal ethanol exposure disrupts intraneocortical circuitry, cortical gene expression, and behavior in a mouse model of FASD. J Neurosci. 2013 Nov;33(48):18893-905.

46 Paus T, Toro R. Could Sex Differences in White Matter be Explained by g ratio? Front Neuroanat. 2009 Sep;3:14.

47 Chomiak T, Hu B. What is the optimal value of the g-ratio for myelinated fibers in the rat CNS? A theoretical approach. PLoS One. 2009 Nov;4(11):e7754.

48 Hüppi PS, Dubois J. Diffusion tensor imaging of brain development. Semin Fetal Neonatal Med. 2006 Dec;11(6):489-97.

49 Dudink J, Lequin M, van Pul C, Buijs J, Conneman N, van Goudoever J, et al. Fractional anisotropy in white matter tracts of very-low-birth-weight infants. Pediatr Radiol. 2007 Dec;37(12):1216-23.

50 Cheong JL, Thompson DK, Wang HX, Hunt RW, Anderson PJ, Inder TE, et al. Abnormal white matter signal on MR imaging is related to abnormal tissue microstructure. AJNR Am J Neuroradiol. 2009 Mar;30(3):623-8.

51 Counsell SJ, Allsop JM, Harrison MC, Larkman DJ, Kennea NL, Kapellou O, et al. Diffusion-weighted imaging of the brain in preterm infants with focal and diffuse white matter abnormality. Pediatrics. 2003 Jul;112(1 Pt 1):1-7.

52 Back SA, Han BH, Luo NL, Chricton CA, Xanthoudakis S, Tam J, et al. Selective vulnerability of late OL progenitors to hypoxia-ischemia. J Neurosci. 2002 Jan;22(2):455-63.

53 Zhou J, Butler EE, Rose J. Neurologic correlates of gait abnormalities in cerebral palsy: implications for treatment. Front Hum Neurosci. 2017 Mar;11:103.

54 Wu YW, Day SM, Strauss DJ, Shavelle RM. Prognosis for ambulation in cerebral palsy: a population-based study. Pediatrics. 2004 Nov;114(5):1264-71.

55 Clowry GJ, Basuodan R, Chan F. What are the Best Animal Models for Testing Early Intervention in Cerebral Palsy? Front Neurol. 2014 Dec;5:258. 